Pathologe 2010 - [Suppl 2] 31:334-335

DOI 10.1007/s00292-010-1381-3

C) Springer-Verlag 2010

\section{Satzung der Deutschen Gesellschaft für Pathologie e. V.}

§ 1 Name, Sitz und Rechtsfähigkeit. Der Name der Gesellschaft lautet „Deutsche Gesellschaft für Pathologie e. V.“. Ihr Sitz ist Göttingen. Sie ist in das dortige Vereinsregister eingetragen.

§ 2 Zweck der Gesellschaft. Zweck der Gesellschaft ist: Förderung der wissenschaftlichen und ärztlichen Belange der Pathologie im weitesten Umfang in dem Bestreben, der Erforschung und Abwehr von Krankheiten zu dienen und die $\mathrm{Pa}$ thologie in ihrer zentralen Bedeutung für die gesamte Medizin weiterzuentwickeln. Hierzu dienen: Die Abhaltung einer Jahrestagung und ggf. weiterer Tagungen und die Veröffentlichung der Referate in einer geeigneten Form. Hierzu dienen weiterhin der Gedanken- und Erfahrungsaustausch zwischen Pathologen; die Herstellung und Vertiefung der Beziehungen zu den der Pathologie verbundenen Disziplinen der Medizin und der Naturwissenschaften sowie zu in- und ausländischen Fachgesellschaften; die Auszeichnung von Personen, die sich um die Entwicklung der Pathologie besonders verdient gemacht haben (Rudolf-Virchow-Medaille), die Auszeichnung wissenschaftlicher Arbeiten auf dem Gebiet der Pathologie (Rudolf-VirchowPreis), die Arbeitsgemeinschaften der Gesellschaft und die Förderung des wissenschaftlichen Nachwuchses.

§ 3 Gemeinnützigkeit. 1. Die Deutsche Gesellschaft für Pathologie verfolgt ausschließlich und unmittelbar gemeinnützige Zwecke im Sinne des Abschnitts „Steuerbegünstigte Zwecke“ der Abgabeordnung. 2. Die Gesellschaft ist selbstlos tätig. Sie verfolgt nicht in erster Linie eigenwirtschaftliche Zwecke. 3. Mittel der Gesellschaft dürfen nur für Satzungszwecke verwendet werden. Die Mitglieder erhalten keine Zuwendungen aus Mitteln der Gesellschaft. 4. Es darf keine Person durch Ausgaben, die dem Zweck der Gesellschaft fremd sind oder durch unverhältnismäßig hohe Vergütungen begünstigt werden. 5. Bei Auflösung oder Aufhebung der Gesellschaft oder bei Wegfall ihres steuererbegünstigen Zweckes fällt das Vermögen an die Deutsche Forschungsgemeinschaft (DFG Bonn-Bad Godesberg), die es ausschließlich für gemeinnützige Zwecke (insb. zur Förderung der Wissenschaft und Forschung) zu verwenden hat.

§ 4 Mitgliedschaft. 1. Die Deutsche Gesellschaft für Pathologie hat ordentliche Mitglieder, außerordentliche Mitglieder, Ehrenmitglieder, korrespondierende Mitglieder und fördernde Mitglieder. 2. Ordentliche Mitglieder können Ärzte, Zahnärzte, Tierärzte sowie Naturwissenschaftler mit abgeschlossener Hochschulausbildung werden. Über die Aufnahme entscheidet der Vorstand. 3. Der Jahresbeitrag für die ordentlichen Mitglieder wird von der Mitgliederversammlung für das folgende Geschäftsjahr festgesetzt. Jedes beitragespflichtige Mitglied ist zur Zahlung des Beitrages zu Anfang des laufenden Jahres verpflichtet. Sonderregelungen ergeben sich aus der jährlich neu beschlossenen Beitragesordnung. Ordentliche Mitglieder können nach Beendigung ihrer beruflichen Tätigkeit auf Antrag außerordentliche Mitglieder werden. Au- ßerordentliche Mitglieder erhalten Sonderkonditionen für den Mitgliedsbeitrag (Reduktion oder Freistellung), die von der Mitgliederversammlung festgelegt werden, und Einschränkungen bei den Aussendungen der Gesellschaft zur Folge haben. Sie werden im Mitgliedsverzeichnis geführt und können zu den Bedingungen ordentlicher Mitglieder an Veranstaltungen der Gesellschaft teilnehmen. Sie haben nicht mehr das Stimm- und Wahlrecht. Fördernde Mitglieder zahlen neben dem Förderbeitrag keinen Jahresbeitrag oder eine Umlage. Jedes ordentliche und jedes fördernde Mitglied ist zur Zahlung des Jahresbeitrages bzw. Förderbeitrages am Anfang des laufenden Jahres verpflichtet. Sonderregelungen können sich aus der jährlich neu beschlossenen Beitragesordnung ergeben. Die Mitgliederversammlung kann eine Umlage beschließen. 4. Die Mitgliedschaft endet durch Austritt, Erlöschen, Ausschluss oder durch den Tod. Die Mitgliedschaft kann jederzeit zum Jahresende durch Austrittserklärung schriftlich gekündigt werden. Gezahlte Mitgliedsbeiträge und erhobene Umlagen werden nicht zurückerstattet. Nichtbezahlung des Beitrages trotz dreifacher Mahnung führt zum Erlöschen der Mitgliedschaft. Dies wird dem Mitglied bekannt gegeben. Der Ausschluss eines Mitglieds kann vom Vorstand nach Anhörung des betreffenden Mitglieds verfügt werden, wenn es die Interessen der Gesellschaft schwerwiegend geschä-

Gemäß Beschluss der Mitgliederversammlung vom 31. Mai 2007 
digt hat. Gegen den Ausschluss ist die Beschwerde zulässig, die innerhalb eines Monats nach Zustellung des Ausschlussbescheides beim Vorstand einzulegen ist. Über die Beschwerde entscheidet die Mitgliederversammlung mit einfacher Mehrheit. Der Ausschluss und das Erlöschen der Mitgliedschaft werden mit Zustellung des Ausschlussbescheides wirksam. 5. Die ordentlichen Mitglieder sind stimmberechtigt und wählbar. 6. Mitglieder oder Persönlichkeiten, die sich um die Pathologie besondere Verdienste erworben haben, können von der Mitgliederversammlung zu Ehrenmitgliedern ernannt werden. Ehrenmitglieder zahlen keinen Jahresbeitrag. 7. Die Mitgliederversammlung kann vom Vorstand vorgeschlagene, nicht dem deutschen Sprachraum angehörende Wissenschaftler zu korrespondierenden Mitgliedern der Gesellschaft wählen. Korrespondierende Mitglieder zahlen keinen Jahresbeitrag. 8. Personen sowie private und öffentliche Vereinigungen, die die Ziele der Gesellschaft unterstützen, können vom Vorstand als fördernde Mitglieder aufgenommen werden. Die Höhe des Förderbeitrages wird vom Vorstand im Einvernehmen mit dem fördernden Mitglied festgesetzt. Fördernde Mitglieder sind weder stimmberechtigt noch wählbar.

§ 6 Vorstand. 1. Der Vorstand besteht aus folgenden Mitgliedern: a) dem amtierenden Vorsitzenden (auf 2 Jahre gewählt, zweimalige Wiederwahl in Kontinuität möglich) b) dem Tagungspräsidenten (einmalig für 1 Jahr gewählt, dann Ersatz durch den nach einem Amtsjahr nachrückenden designierten Tagungspräsidenten) c) designierter Tagungspräsident (für 2 Jahre gewählt) d) Geschäftsführendes Vorstandsmitglied (für 3 Jahre gewählt, zweimalige Wiederwahl in Kontinuität möglich) e) sieben Beisitzer mit jeweils einem Vertreter aus Österreich und der Schweiz (Amtszeit 2 Jahre, zweimalige Wiederwahl in Kontinuität möglich). 2. Vorstand im Sinne des $₫ 26$ BGB sind der amtierende Vorsitzende, der Tagungspräsident als stellvertretender Vorsitzender und das Geschäftsführende Vorstandsmitglied. Sie vertreten die Gesellschaft gerichtlich und außergerichtlich. Jeder der genannten Vorstandsmitglieder hat Einzelvertretungsbefugnis. 3. Die
Wahl der unter Abs. 1 Buchstabe a-e genannten Vorstandsmitglieder erfolgt in der ordentlichen Mitgliederversammlung mit einfacher Mehrheit. 4. Der Vorstand gibt sich eine Geschäftsordnung, über die die Mitgliederversammlung mit einfacher Mehrheit beschließt.

§7 Die Mitgliederversammlung. 1. Mindestens einmal im Jahr ist vom Vorstand eine ordentliche Mitgliederversammlung einzuberufen. Die Einladung muss schriftlich mindestens 4 Wochen vor dem vorgesehenen Termin erfolgen und die Tagesordnung enthalten. Eine außerordentliche Mitgliederversammlung ist gem. vorstehender Regelung vom Vorstand einzuberufen, wenn das Interesse der Gesellschaft es erfordert oder wenn ein Zehntel der ordentlichen Mitglieder dies schriftlich unter Angabe des Zwecks und der Gründe beantragt. Die Einberufungsfrist kann auf eine Woche abgekürzt werden. In der Mitgliederversammlung sind folgende Angelegenheiten zu behandeln: a) die Wahl von Vorstandsmitgliedern b) Beschlussfassung über Tagungsthemen und -orte c) die Entgegennahme des Finanzberichtes über das abgelaufene Kalenderjahr und die Festsetzung des Mitgliedsbeitrages für das kommende Kalenderjahr d) Entlastung des Vorstandes e) Wahl von zwei Kassenprüfern f) Beschlussfassung über die Ernennung von Ehrenmitgliedern und die Wahl von korrespondierenden Mitgliedern g) Beschlussfassung über Satzungsänderungen. 2. Anregungen und Anträge für die Mitgliederversammlung sind dem Vorstand bis spätestens zwei Wochen vor dem Termin der Mitgliederversammlung schriftlich einzureichen. 3. Die Mitgliederversammlung fasst ihre Beschlüsse grundsätzlich mit der einfachen Mehrheit der anwesenden Mitglieder. Die Auflösung der Gesellschaft kann nur mit einer Dreiviertel-Mehrheit der anwesenden Mitglieder beschlossen werden. Gleiches gilt für eine Änderung von $\$ 2$ und $₫ 3$ der Satzung. Sonstige Satzungsänderungen bedürfen einer Zweidrittel-Mehrheit. Über die Mitgliederversammlung ist ein Protokoll zu führen, das vom amtierenden Vorsitzenden und vom geschäftsführenden Vorstandsmitglied zu unterschreiben ist.
§ 8 Geschäftsjahr, Sonstiges. Das Geschäftsjahr der Gesellschaft ist das Kalenderjahr. Satzungsänderungen gelten ab Eintrag in das Vereinsregister. Vorstandsmitglieder nach $₫ 6$ Abs. 2 sind sofort nach ihrer Wahl im Amt. 